\title{
A wave-bending structure at Ka-band using 3D-printed metamaterial
}

Junqiang Wu, Min Liang, and Hao Xin

Citation: Journal of Applied Physics 123, 124109 (2018); doi: 10.1063/1.5003847

View online: https://doi.org/10.1063/1.5003847

View Table of Contents: http://aip.scitation.org/toc/jap/123/12

Published by the American Institute of Physics

\section{Articles you may be interested in}

Experimental realization of all-angle negative refraction in acoustic gradient metasurface

Applied Physics Letters 111, 221602 (2017); 10.1063/1.5004005

Perspective: Acoustic metamaterials in transition

Journal of Applied Physics 123, 090901 (2018); 10.1063/1.5007682

Manipulating acoustic wave reflection by a nonlinear elastic metasurface

Journal of Applied Physics 123, 124901 (2018); 10.1063/1.5015952

Investigation of acoustic metasurfaces with constituent material properties considered

Journal of Applied Physics 123, 124905 (2018); 10.1063/1.5007863

An optically transparent metasurface for broadband microwave antireflection

Applied Physics Letters 112, 073504 (2018); 10.1063/1.5018017

Graphene-induced modulation effects on magnetic plasmon in multilayer metal-dielectric-metal metamaterial Applied Physics Letters 112, 131101 (2018); 10.1063/1.5022870

\section{PHYSICS TODAY}

MANAGER'S GUIDE

WHITEPAPERS

\section{READ NOW}

PRESENTED BY

Accelerate R\&D with
Multiphysics Simulation 


\title{
A wave-bending structure at Ka-band using 3D-printed metamaterial
}

\author{
Junqiang Wu, Min Liang, and Hao Xin ${ }^{\mathrm{a})}$ \\ Department of Electrical and Computer Engineering, University of Arizona, Tucson, Arizona 85719, USA
}

(Received 7 September 2017; accepted 11 March 2018; published online 30 March 2018)

\begin{abstract}
Three-dimensional printing technologies enable metamaterials of complex structures with arbitrary inhomogeneity. In this work, a $90^{\circ}$ wave-bending structure at the Ka-band $(26.5-40 \mathrm{GHz})$ based on 3D-printed metamaterials is designed, fabricated, and measured. The wave-bending effect is realized through a spatial distribution of varied effective dielectric constants. Based on the effective medium theory, different effective dielectric constants are accomplished by special, 3D-printable unit cells, which allow different ratios of dielectric to air at the unit cell level. In contrast to traditional, metallic-structure-included metamaterial designs, the reported wave-bending structure here is all dielectric and implemented by the polymer-jetting technique, which features rapid, low-cost, and convenient prototyping. Both simulation and experiment results demonstrate the effectiveness of the wave-bending structure. Published by AIP Publishing. https://doi.org/10.1063/1.5003847
\end{abstract}

\section{INTRODUCTION}

Manipulating electromagnetic (EM) wave propagation has attracted researchers' attention because of many exciting potential applications. For instance, a Luneburg lens has a focal point for the incident wave from the opposite side, which lends itself to the microwave direction of arrival estimation. ${ }^{1}$ Frequency selective surfaces ${ }^{2}$ act as band-pass filters or band-stop filters for propagating EM waves and find applications in suppressing the impact of out-of-band energy. Moreover, the EM cloak design is vastly intriguing for concealing an object from detection by EM waves. ${ }^{3}$

Transformation optics (TO), which utilizes metamaterials to achieve EM wave manipulation, has been extensively investigated. ${ }^{4-6}$ However, TO is plagued by its magnetic and anisotropic material requirement, ${ }^{7,8}$ which is difficult to achieve in the experiment. Fortunately, a coordinate mapping within the restricted geometry can transform the anisotropic condition to the isotropic or the quasi-isotropic condition. Several algorithms to achieve such coordinate mapping of TO have been proposed. ${ }^{3,9}$ However, it often requires complicated optimization.

Apart from TO, geometrical optics (GO) has been investigated to control EM wave propagation. The advantage of GO is that it needs nonmagnetic and isotropic materials rather than magnetic and anisotropic materials. ${ }^{10}$ Therefore, GObased structures are easier to realize compared to TO-based structures. A wave bending structure has been designed based on the traditional metallic-structure-based metamaterials. ${ }^{10}$ Nevertheless, fabrication requires a manual assembly of multiple metamaterial layers, which is not convenient enough. The inhomogeneous structure required by genetic algorithms (GA) can be conveniently realized by additive manufacturing (AM) or 3D printing technologies. AM allows arbitrary 3D structures with less expense and more convenient prototyping compared to conventional fabrication technologies. The EM applications of 3D printing include EM bandgap structures, ${ }^{11}$

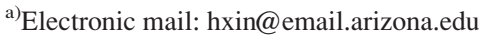

$\mathrm{THz}$ electromagnetic crystal horn antennas, ${ }^{12} \mathrm{THz}$ waveguides, ${ }^{13}$ holograms, ${ }^{14}$ Luneburg lens antennas, ${ }^{15}$ and dielectric reflectarray antennas. ${ }^{16}$

In this work, a metamaterial $90^{\circ}$ wave-bending structure at the Ka-band $(26.5 \mathrm{GHz}-40 \mathrm{GHz})$ based on $\mathrm{GO}$ is designed. The metamaterial wave-bending structure is compatible with 3D printing technologies, which provides a less-expensive and more-convenient-prototyping solution compared to metallic-structure-included wave-bending structures. ${ }^{10}$ The dielectric constant spatial distribution is calculated according to the Eikonal equation. Different effective dielectric constants are achieved based on the effective medium theory by varying the ratio of the $3 \mathrm{D}$-printed polymer material $\left(\varepsilon_{r}=2.7\right)$ to air $\left(\varepsilon_{r}=1\right)$ at the unit cell level. The finally designed wave-bending structure is printed using a polymer jetting 3D printer. ${ }^{15}$ Both simulation and experimental results prove the effectiveness of the wave-bending structure. This work unveils the promising potential of $3 \mathrm{D}$ printing technologies for implementation of inhomogeneous metamaterials for a number of EM applications. It may also be inspiring for other applications. For example, a commercial $60 \mathrm{GHz}$ rectangular waveguide bend can be expensive. A $3 \mathrm{D}$-printed dielectric waveguide bend is promising as a lower-cost alternative due to its inexpensive material cost and simplified fabrication process. The cost may be two orders of magnitude lower. Besides, 3D-printed wave-bending structures are interesting that they may shed some light on more advanced designs including electromagnetic cloaking devices, as the bending wave is the first step to be "invisible."17

\section{DESIGN}

GO is the optics neglecting the wavelength, which is applicable when the wavelength is much smaller compared to the size of structures. At microwave frequencies, the wavelength is much larger and often comparable with the microwave components. GO, however, even for structures of a few wavelengths, can still produce meaningful and useful 


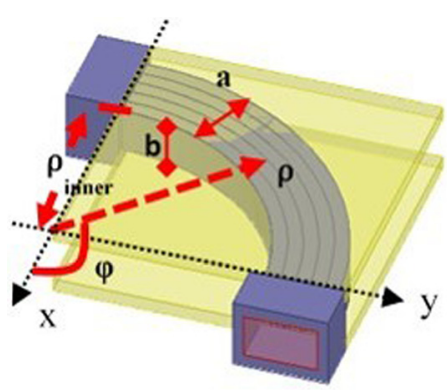

(a)

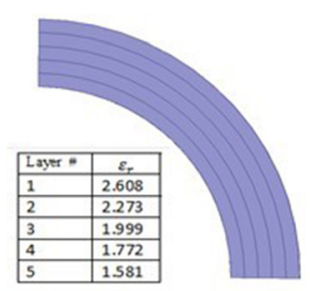

(b)

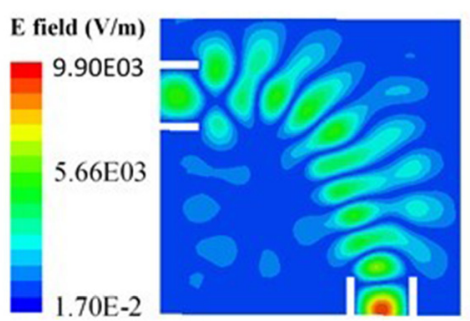

(c)

FIG. 1. (a) HFSS model of the ideal wave-bending structure (ideal model). (b) Dielectric constant distribution of the bend. (c) The simulated $E$ field distribution at $30 \mathrm{GHz}$ of the model in (a).

results. ${ }^{18}$ Derived from GO, the Eikonal equation ${ }^{19}$ describes the relationship between the optical/EM wave path $S$ and the refractive index $n$ in a $2 \mathrm{D}$ plane (cylindrical coordinate system).

$$
|\nabla S(\rho, \varphi)|^{2}=n^{2}
$$

where $\rho$ is the radius, $\varphi$ is the azimuth angle, and $n=\sqrt{\mu_{r} \epsilon_{r}}$.

In a wave-bending structure, $S$ can be defined as ${ }^{10}$

$$
S=A \varphi+C,
$$

where $\mathrm{A}$ and $\mathrm{C}$ are two constants and $\varphi$ is the central angle corresponding to the bend arc. Substituting (2) into (1), the relationship between the dielectric constant $\varepsilon_{\mathrm{r}}$ and radius $\rho$ can be obtained

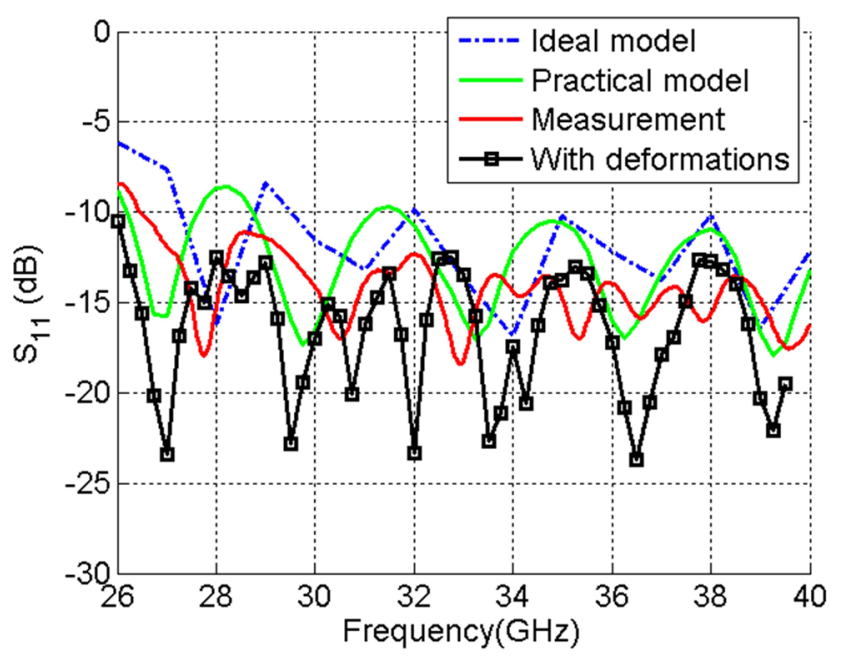

$$
\begin{gathered}
|\nabla S|^{2}=\left|\overrightarrow{a_{\rho}} \frac{\partial S}{\partial \rho}+\overrightarrow{a_{\varphi}} \frac{1}{\rho} \frac{\partial S}{\partial \varphi}+\overrightarrow{a_{z}} \frac{\partial S}{\partial z}\right|=\left(\frac{A}{\rho}\right)^{2}=n^{2}, \\
\varepsilon_{r}=(A / \rho)^{2} \quad \text { when } \mu_{r}=1 .
\end{gathered}
$$

That is to say, $\varepsilon_{\mathrm{r}}$ distribution is determined by $A$ and $\rho$.

In this work, $A=0.0323$. The inner radius of the wavebending structure is $\rho_{\text {inner }}=20 \mathrm{~mm}$. Two practical factors are considered to opt for the appropriate $\mathrm{A}$ and $\rho$ in this design. First, the wave-bending structure should have minimal dielectric loss. The 3D printing material to be utilized, VeroBlackPlus RGD875 from Stratasys Ltd., has $\tan \delta$ $=0.02 .{ }^{15}$ Small radius $\rho$ means a short wave path and thus small dielectric loss. Therefore, the radius should be as small as possible. Second, the $\varepsilon_{\mathrm{r}}$ value should be between 1 and $2.7{ }^{15}$ which is readily realizable with our polymer jetting rapid prototyping printer. The width of the wave-bending structure is $a=7.112 \mathrm{~mm}$, in accordance with the standard WR-28 rectangular waveguide width. Correspondingly, the ANSYS high frequency structure simulator (HFSS) model of the ideal wave-bending structure is depicted in Fig. 1(a). Five layers are used in the design, with the calculated $\varepsilon_{\mathrm{r}}$ of each layer displayed in Fig. 1(b). The wave-bending structure has the largest dielectric constant $\varepsilon_{\mathrm{r}}=2.608$ for layer \#1 and the smallest dielectric constant $\varepsilon_{\mathrm{r}}=1.581$ for layer \#5. The overall wave-bending structure is about $35 \mathrm{~mm}$ long, which is roughly $3.5 \lambda_{0}$ at $30 \mathrm{GHz}$. In consideration of the experiment, the height of the structure is set as $b=3.556 \mathrm{~mm}$. Consequently, the wave-bending structure has compatible interfaces with the standard WR-28 rectangular waveguide. Perfect electric conductor (PEC) boundaries are added on top and bottom of the ideal wave-bending structure. Two WR-28 waveguides are employed at two ends of the wave-bending structure as feeds. Figure 1(c) shows the simulated E-field distribution at $30 \mathrm{GHz}$, which clearly demonstrates the effectiveness of the wave-bending structure. The simulated Sparameters are shown in Fig. 2 (blue solid lines). $S_{21}$ is about $-2 \mathrm{~dB}$ over the frequency band. The wave-bending phenomenon of the designed all-dielectric metamaterial structure is obvious. It can be noted that there is some leakage out of the

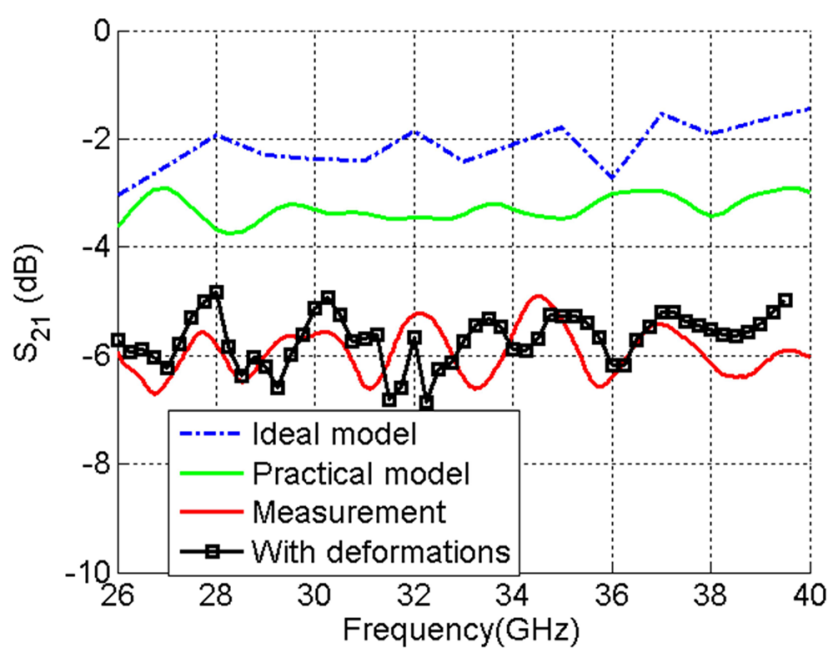

FIG. 2. S-parameters of the ideal model [Fig. 1(a); dashed blue lines], practical model [Fig. 4(b); solid green lines], measurement (solid red lines), and simulation after adding imperfections into the model (square markers). 
wave-bending structure in Fig. 1(c). The leakage stems from two reasons. First, the size of the bend is comparable with the wavelength rather than much larger than the wavelength as is assumed by GO approximation. Second, the wave-bending structure is divided into only a limited number of layers in the $\rho$ direction owing to compromise in fabrication. If more layers thus smoother $\varepsilon_{\mathrm{r}}$ variation is employed, less leakage is expected.

The desired $\varepsilon_{\mathrm{r}}$ distribution is realized using unit cell structures in experiment. Each unit cell contains two types of materials, the 3D-printed polymer and air. The 3D-printed polymer is VeroBlackPlus RGD875 with $\varepsilon_{\mathrm{r}}=2.7$, while air has $\varepsilon_{\mathrm{r}}=1$. Based on the effective medium theory, different effective dielectric constants between 1 and 2.7 can be achieved simply by adjusting the filling ratio of the polymer material in the unit cell (i.e., mixing polymer and air at different ratios). That is to say, the mixture of the two is capable of realizing the desired $\varepsilon_{\mathrm{r}}$ of layers \#1-\#5.

Arc-shaped unit cells are ideal candidates to fit the shape of the wave-bending structure. However, when such unit cells are small enough, they resemble cuboid unit cells. Consequently, the cuboid unit cell, e.g., Fig. 3(a), as an approximation to the arc shaped unit cell, is used for easier analysis. The unit cell has a radial size in the $\rho$ direction of $1.4224 \mathrm{~mm}$ and an angular size in the $\varphi$ direction of $\pi \rho / 50$. The radius and the unit cell dimension in the $\varphi$ direction for each layer are illustrated in Table I. The largest unit cell is $1.52 \mathrm{~mm}$ along the $\varphi$ direction, which is about $0.2 \lambda_{0}$ at $40 \mathrm{GHz}$ and in turn, leading to a reasonable condition for the effective medium approximation.

Three types of unit cell structures, as shown in Fig. 3, are employed in the design to make sure that the structure feature size (minimum gap and structure size) is larger than $0.3 \mathrm{~mm}$ for reasonable printing quality while achieving the desired $\varepsilon_{\mathrm{r}}$. The blue part is the 3D-printed polymer material, which is placed in a transparent air box. Unit cell A in Fig. 3 (a) is used for layer \#1. The slab along the $\varphi$ direction helps maintain a high polymer filling ratio $f$ and thereby high effective relative permittivity $\varepsilon_{\mathrm{r}}$ of the unit cell, while keeping larger-than $0.3 \mathrm{~mm}$ gaps formed by neighbor unit cells along the $\varphi$ direction. Unit cell B in Fig. 3(b) is for layers $\# 2$, \#3, and \#4 with only the slab along the $\rho$ direction utilized. Unit cell C in Fig. 3(c) is for layer \#5. With only the slab in the $\varphi$ direction, Unit cell $\mathrm{C}$ guarantees that the minimum structure is larger than $0.3 \mathrm{~mm}$ for small $\varepsilon_{\mathrm{r}}$. The approximated permittivity of a unit cell is calculated by the analytical effective medium approximation using $\varepsilon_{r}=\varepsilon_{p} \cdot f+1 \cdot(l-f)$, where $\varepsilon_{p}$ is the relative permittivity of the polymer material and $f$ is the volume ratio of the 3Dprinted polymer in the unit cell. ${ }^{15}$ Long wave approximation
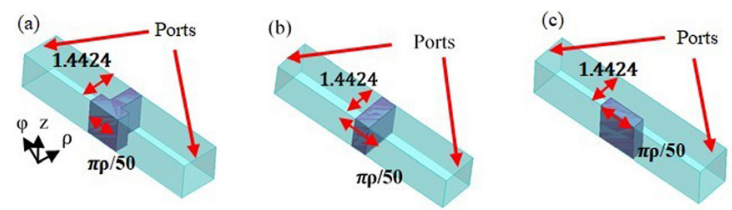

Unit: $\mathrm{mm}$

FIG. 3. The effective dielectric constant simulation setup in HFSS for unit cells (a) A, (b) B, and (c) C.
TABLE I. Unit cell dimension in $\varphi$ and realized $\varepsilon_{\text {eff }}$ in terms of layer \#.

\begin{tabular}{lccccc}
\hline \hline Layer \# & 1 & 2 & 3 & 4 & 5 \\
\hline$\rho(\mathrm{mm})$ & 20 & 21.4224 & 22.8448 & 24.2672 & 25.6896 \\
Dimension in $\varphi$ & 1.2566 & 1.3460 & 1.4354 & 1.5243 & 1.5 \\
direction $(\mathrm{mm})$ & & & & & 1.586 \\
Realized $\varepsilon_{\mathrm{eff}}$ & 2.626 & 2.295 & 1.956 & 1.758 & 1.58 \\
\hline \hline
\end{tabular}

is also commonly applied to describe and predict material's electromagnetic properties in the microwave frequency range. ${ }^{20}$ A simple form could be $n_{r}=n_{p} \cdot f+l \cdot(1-f) .{ }^{21}$ For this specific application, both approximations yield to similar effective dielectric constants $(\Delta= \pm 0.3)$. Next, the calculated effective permittivity is verified by finite-element simulations using HFSS models, as plotted in Fig. 3. In the models, the 3D-printed polymer unit-cell structures are placed in an air box, where PEC (top and bottom) and perfect magnetic conductor (sides) boundaries are used. Transverse electromagnetic wave ports are at two ends of the air box, and deembedding is done to obtain the $S$-parameters of the unit cells. $\varepsilon_{\text {eff }}$ can then be extracted based on the Nicholson-Ross-Weir method. ${ }^{22,23}$ The extracted $\varepsilon_{\text {eff }}$ is listed in Table I. The dimensions of unit cells for each layer are shown in Fig. 4(a).

The complete wave-bending structure is generated by combining all unit cell structures. The HFSS simulation for the entire structure is performed as depicted in Fig. 4(b). Two $33 \mathrm{~mm} \times 33 \mathrm{~mm}$ copper plates are placed on top and bottom of the wave-bending structure. The copper plates imitate the PEC boundary conditions on top and bottom. The wave-bending structure is fed by two WR- 28 rectangular waveguides. Metal flanges for waveguide connection are included as well.

Figure 4(c) illustrates the $E$ field distribution at $30 \mathrm{GHz}$. The simulated frequency response of this HFSS model is given in Fig. 2. The $S_{11}$ of the wave-bending structure is smaller than $-10 \mathrm{~dB}$ at most frequencies. $S_{21}$ is about $-3.5 \mathrm{~dB}$ within the Ka-band, which is about $1.5 \mathrm{~dB}$ smaller than the ideal model's $S_{21}$. The extra loss is due to the dielectric loss of the 3D-printable material $(\tan \delta=0.02)$. As a

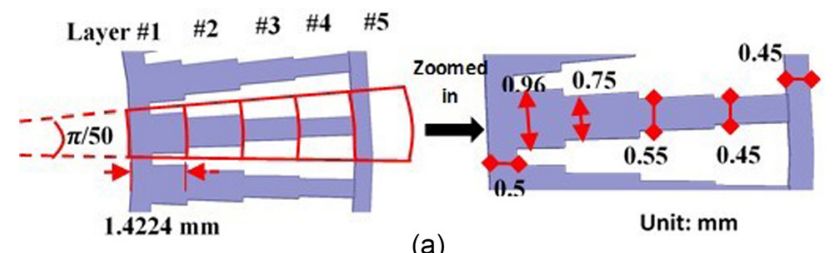

(a)
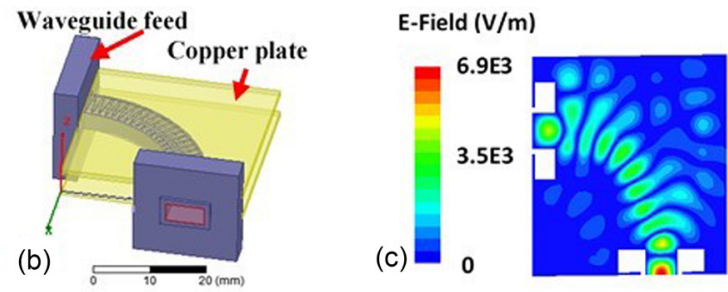

FIG. 4. (a) The dimensions of unit cell structures. (b) The HFSS model of the wave-bending structure (practical model). (c) The $E$ field distribution at $30 \mathrm{GHz}$. 
(a)

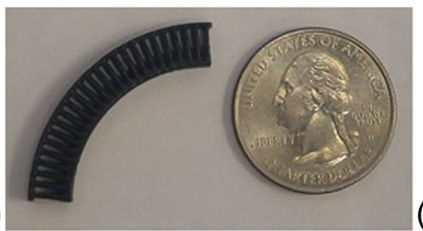

(b)

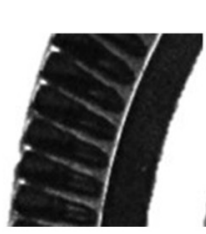

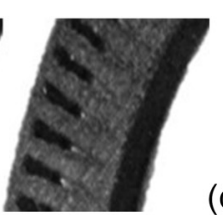

(c)

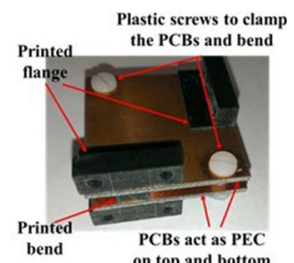

FIG. 5. (a) Photograph of the 3D-printed wave-bending structure. (b) Top zoom-in view (left) and bottom zoom-in view (right) of the wave-bending structure; the gaps on top are larger and the gaps at the bottom are smaller or even merged, showing the deformation of the wave-bending structure. (c) Experimental setup of the wave-bending structure.

result, the simulation corroborates the effectiveness of the wave-bending structure.

\section{FABRICATION AND MEASUREMENT}

The designed wave-bending structure is printed using a polymer jetting rapid prototyping machine, Objet Eden 350 . The printer has a drop-let size of $84 \mu \mathrm{m} \times 42 \mu \mathrm{m} \times 16 \mu \mathrm{m}$. The process includes computer-aided design (CAD) file conversion and structure printing. First of all, the CAD file of a designed 3D object is converted into a series of layered slices, with each slice representing a $16 \mu \mathrm{m}$ thick region of the designed model. Upon receiving the data, the printer head deposits a thin layer of the polymer structure corresponding to the shape that the data describe. Then, the material is cured immediately by the ultraviolet lamps on the print heads. After one layer is finished, the construction stage moves down $16 \mu \mathrm{m}$, and another layer is deposited on top. Layer by layer, the designed 3D object is printed. ${ }^{15}$

The fabricated wave-bending structure is shown in Fig. 5(a). Zoom-in views of the top and bottom are shown in Fig. 5(b). It is observed that the structure has a bit deformation. In contrast to the designed model, air gaps on top slightly expand, while air gaps at the bottom shrink. The reason is that the support material $(\tan \delta=0.1)$ is not used in the printing process. Despite the fact that the water-soluble support material can fill gaps during printing to avoid deformation and afterward be removed by pressured water, ${ }^{15}$ its residue on the structure surface may result in a larger dielectric loss. Consequently, the support material is avoided in the printing process. Inevitably, the polymer tends to droop due to gravity without the support material.

Figure 5(c) shows the experimental setup. Two pieces of printed circuit board (PCBs) with full copper cladding are cut into $33 \mathrm{~mm} \times 33 \mathrm{~mm}$ to apply PEC boundary conditions on top and bottom of the wave-bending structure. To mechanically secure the wave-bending structure, two plastic screws are installed through the PCB boards at the diagonal corners. The screws are distant from the wave-bending structure and have little impact on the propagating EM wave. Four pieces of polymer flanges are printed and mounted on the PCBs for waveguide connections.

The solid red curves in Fig. 2 are the measured $S$-parameters. It is evident that the measurement results are consistent with the simulation results. However, there are some discrepancies. The measured $S_{11}$ is about $2 \mathrm{~dB}$ less than the simulation in average. The measured $S_{21}$ is about $2.5 \mathrm{~dB}$ less than the simulation. As a result, the measured power loss is $\sim 72 \%$ in comparison to the simulated $\sim 50 \%$. The discrepancy arises from two imperfections. First, as aforementioned in Fig. 5(b), the fabricated wave-bending structure experiences some deformation. Second, there are openings between the feeding waveguides and the PCBs during measurement, as demonstrated in Fig. 6(a). Two possible causes lead to the openings. (1) The 3D-printed flanges in Fig. 5(c) are manually glued to the PCBs with certain misalignment. In consequence, the 3D-printed flanges, when connected with the feeding waveguide flanges, may create openings between the PCBs and the feeding waveguides; (2) the two PCBs are not perfectly identical and well aligned, which also contribute to the openings between PCBs and the feeding waveguides after assembly.

To investigate the impact of the above two factors, simulations are carried out with the imperfections, including the above deformation and opening. First, openings are added between the PCB boards and the feeding waveguides in the HFSS model. A $0.5 \mathrm{~mm}$ opening is added between the top PCB and one waveguide. Symmetrically, another $0.5 \mathrm{~mm}$ opening is added between the bottom PCB and the other waveguide, as shown in Fig. 6(a). Moreover, the wavebending structure is divided into 3 layers in the $z$ direction to include the deformation in the model. To mimic the dripping of the printed material, the top layer slab thickness is reduced: the $\varphi$ direction slab thickness is reduced by $0.3 \mathrm{~mm}$ and the $\rho$ direction slab thickness is reduced by $\pi \rho / 360$. The middle layer slab thickness is kept unchanged. The bottom layer slab thickness increases corresponding to the material reduction of the top layer. That is to say, the $\varphi$ direction slab (a)

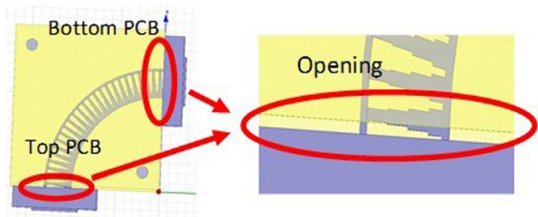

(b)

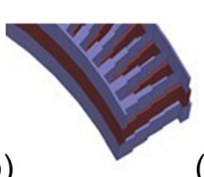

(c)

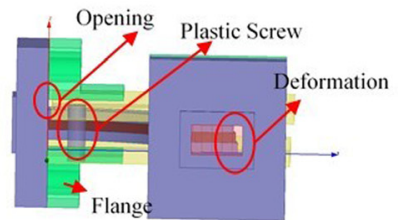

FIG. 6. HFSS models showing (a) the openings on top and bottom, (b) the added deformation of the wave-bending structure, and (c) the final simulation setup with the openings and bend deformation. 
thickness increases by $0.3 \mathrm{~mm}$ and the $\rho$ direction slab thickness increases by $\pi \rho / 360$. The deformation in the HFSS model is depicted in Fig. 6(b). Figure 6(c) presents the HFSS model with the above changes, i.e., with imperfections. The simulation results (square markers) are shown in Fig. 2. A good resemblance between simulation and measurement results is observed for both $S_{11}$ and $S_{21}$. The fact that the simulation and the experiment are not perfectly matched is understandable because the opening size and degree of deformation in the HFSS model are based on estimation and may not be exactly the same as the real physical imperfections. For example, in Fig. 6(b), the bending structure is divided into 3 layers (i.e., top, middle, and bottom), reduced the top layer material, and increased the bottom layer material based on visual estimation. For the practical sample, however, the deformation is continuous from top to bottom rather than 3 discrete layers. Consequently, the exact shape of the practical sample is not what it is in the model. The same is for the openings between PCBs and the feeding waveguides. Therefore, the two curves do not agree exactly with each other. Nevertheless, the simulation shows that those deformations that exist in the practical sample will degrade $S_{21}$ as observed in the experiment and reach a much better agreement with the experiment. The $E$ field distribution at $30 \mathrm{GHz}$ of the HFSS model in Fig. 6(c) is shown in Fig. 7. It shows that the $E$ field strength is smaller at the output end of the wave bending structure in Fig. 7 than that in Fig. 4(c).

Other possible differences between the simulation and experimental setup, including the drilled holes on PCBs, the plastic screws, and the waveguide alignment error, are also simulated separately in HFSS. The simulation results indicate that these factors have a very limited impact on the wave-bending structure performance compared to the aforementioned two factors. Consequently, the structure's deformation and openings between PCBs and waveguides are the culprits of the discrepancy.

To improve the performance of the sample in the future, a support material can be used to reduce deformation. To fully remove the support material on the surface of the model without damaging it, the model may be water-bathed in an ultrasonic cleaner for about one hour. Then, the sample should be air dried for about 2 days to make sure that the moisture is all gone. Besides, to eliminate the openings

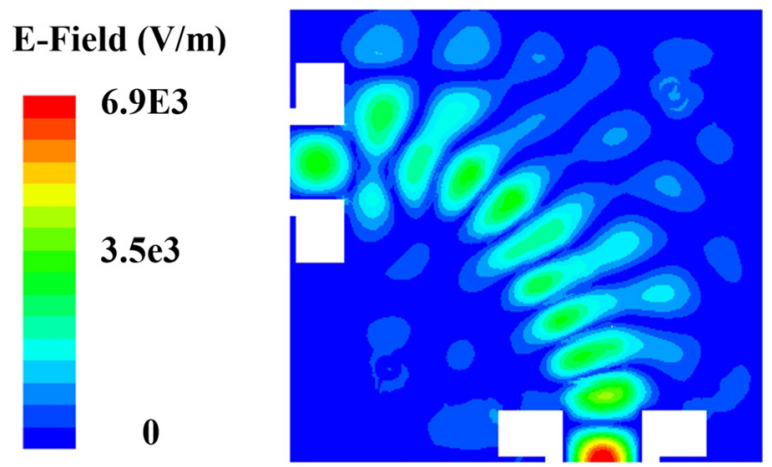

FIG. 7. The $E$ field distribution at $30 \mathrm{GHz}$ of the HFSS model with the openings and bend deformation [Fig. 6(c)]. between PCBs and the feeding waveguides, the test fixture including the board and the flange can be 3D-printed together after which copper plating is used for the surface. This makes sure that no misalignment will be involved in the experiment.

\section{CONCLUSION}

In conclusion, a metamaterial Ka-band $90^{\circ}$ wavebending structure made of dielectric is designed, fabricated, and measured. Compared with its metallic-structure-included metamaterial counterparts, this all-dielectric structure allows lower-cost and faster-prototyping due to its compatibility with the 3D printing technique. The wave-bending effect is achieved through the spatial distribution of different dielectric constants designed using the geometrical optics method. The wave-bending structure is fabricated by the polymer jetting rapid prototyping technique. Both the simulation and experiment results show the effectiveness of the wavebending structure. The experiment results have some discrepancies compared to the simulation results. The analysis shows that the discrepancy is mainly ascribed to the fabrication imperfections, namely, the wave-bending structure's deformation and the openings between PCBs and waveguides. The design method can be extended to other frequency ranges by scaling the unit cell structure dimensions. For example, to extend the design to the X-band, a scale factor of 3.2 is needed based on the standard rectangular waveguide cross-section dimension ratio. Next, the size of the wave-bending structure along with the unit cell structure should increase by 3.2 times. This work suggests that $3 \mathrm{D}$ printing technologies are promising solutions to realize inhomogeneous metamaterials that can control and manipulate the propagation of EM waves effectively. Moreover, 3D printing technologies could also provide a fertile ground for more EM designs embracing inhomogeneity such as wave collimators and beam shifters.

\section{ACKNOWLEDGMENTS}

This work was supported in part by funding from the National Science Foundation under Award No. 1408271.

${ }^{1}$ M. Liang, X. Yu, R. Sabory-Garcia, W.-R. Ng, M. E. Gehm, and H. Xin, in 2012 IEEE/MTT-S International Microwave Symposium Digest (2012), pp. 1-3.

${ }^{2}$ I. M. Ehrenberg, S. E. Sarma, and B. Wu, in 2012 IEEE Antennas and Propagation Society International Symposium, APSURSI (2012), pp. 1-2.

${ }^{3}$ R. Liu, C. Ji, J. J. Mock, J. Y. Chin, T. J. Cui, and D. R. Smith, Science 323, 366 (2009).

${ }^{4}$ J. B. Pendry, D. Schurig, and D. R. Smith, Science 312, 1780 (2006).

${ }^{5}$ Y. Lai, J. Ng, H. Chen, D. Han, J. Xiao, Z.-Q. Zhang, and C. T. Chan, Phys. Rev. Lett. 102, 253902 (2009).

${ }^{6}$ A. Greenleaf, Y. Kurylev, M. Lassas, and G. Uhlmann, Phys. Rev. Lett. 99, 183901 (2007).

${ }^{7}$ N. B. Kundtz, D. R. Smith, and J. B. Pendry, Proc. IEEE 99, 1622 (2011).

${ }^{8}$ Z. L. Mei and T. J. Cui, J. Appl. Phys. 105, 104913 (2009).

${ }^{9}$ L. H. Gabrielli, D. Liu, S. G. Johnson, and M. Lipson, Nat. Commun. 3, 1217 (2012)

${ }^{10}$ Z. L. Mei and T. J. Cui, Opt. Express 17, 18354 (2009).

${ }^{11}$ Z. Wu, J. Kinast, M. E. Gehm, and H. Xin, Opt. Express 16, 16442 (2008).

${ }^{12}$ Z. Wu, M. Liang, W.-R. Ng, M. Gehm, and H. Xin, IEEE Trans. Antennas Propag. 60, 5557 (2012).

${ }^{13}$ Z. Wu, W.-R. Ng, M. E. Gehm, and H. Xin, Opt. Express 19, 3962 (2011). 
${ }^{14}$ W.-R. Ng, D. R. Golish, H. Xin, and M. E. Gehm, Opt. Express 22, 3349 (2014).

${ }^{15}$ M. Liang, W.-R. Ng, K. Chang, K. Gbele, M. E. Gehm, and H. Xin, IEEE Trans. Antennas Propag. 62, 1799 (2014).

${ }^{16}$ P. Nayeri, M. Liang, R. Sabory-Garcia, M. Tuo, F. Yang, M. Gehm, H. Xin, and A. Elsherbeni, IEEE Trans. Antennas Propag. 62, 2000 (2014).

${ }^{17}$ X. Ni, Z. J. Wong, M. Mrejen, Y. Wang, and X. Zhang, Science 349, 1310 (2015).

${ }^{18}$ F. S. Holt, Application of Geometrical Optics to the Design and Analysis of Microwave Antennas (DTIC Document, 1967).
${ }^{19}$ M. Born and E. Wolf, Principles of Optics: Electromagnetic Theory of Propagation, Interference and Diffraction of Light (Cambridge University Press, 1999), p. 117.

${ }^{20}$ D. Bychanok, S. Li, G. Gorokhov, K. Piasotski, D. Meisak, P. Kuzhir, E. A. Burgess, C. P. Gallagher, F. Y. Ogrin, A. P. Hibbins, A. Pasc, A. SanchezSanchez, V. Fierro, and A. Celzard, J. Appl. Phys. 121, 165103 (2017).

${ }^{21}$ D. G. Stavenga, S. Foletti, G. Palasantzas, and K. Arikawa, Proc. R. Soc. London, Ser. B 273, 661 (2006).

${ }^{22}$ A. M. Nicolson and G. F. Ross, IEEE Trans. Instrum. Meas. 19, 377 (1970).

${ }^{23}$ W. B. Weir, Proc. IEEE 62, 33 (1974). 